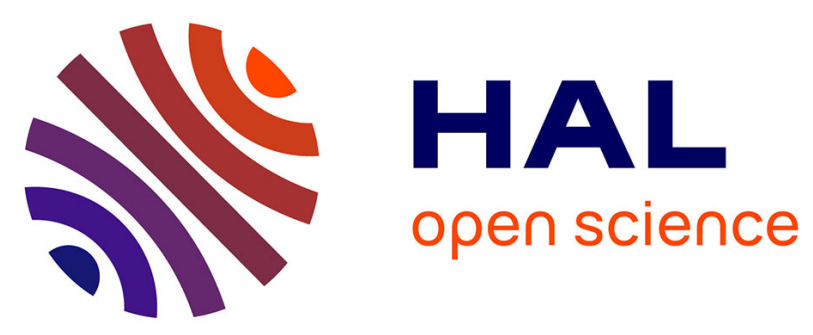

\title{
Resonant Faraday effect using high-order harmonics for the investigation of ultrafast demagnetization
}

\author{
Carla Alves, Guillaume Lambert, Victor Malka, Michel Hehn, Grégory \\ Malinowski, Marcel Hennes, Valentin Chardonnet, Emmanuelle Jal, Jan \\ Lüning, Boris Vodungbo
}

\section{To cite this version:}

Carla Alves, Guillaume Lambert, Victor Malka, Michel Hehn, Grégory Malinowski, et al.. Resonant Faraday effect using high-order harmonics for the investigation of ultrafast demagnetization. Physical Review B: Condensed Matter and Materials Physics (1998-2015), 2019, 100 (14), 10.1103/PhysRevB.100.144421 . hal-02337593

\section{HAL Id: hal-02337593 \\ https://hal.univ-lorraine.fr/hal-02337593}

Submitted on 29 Oct 2019

HAL is a multi-disciplinary open access archive for the deposit and dissemination of scientific research documents, whether they are published or not. The documents may come from teaching and research institutions in France or abroad, or from public or private research centers.
L'archive ouverte pluridisciplinaire HAL, est destinée au dépôt et à la diffusion de documents scientifiques de niveau recherche, publiés ou non, émanant des établissements d'enseignement et de recherche français ou étrangers, des laboratoires publics ou privés. 


\title{
Resonant Faraday effect using high-order harmonics for the investigation of ultrafast demagnetization
}

\author{
Carla Alves, Guillaume Lambert, ${ }^{*}$ and Victor Malka \\ Laboratoire d'Optique Appliquée, ENSTA ParisTech, CNRS, École Polytechnique, Institut Polytechnique de Paris, \\ 828 boulevard des Maréchaux, Palaiseau Cedex 91762, France \\ Michel Hehn and Gregory Malinowski \\ Institut Jean Lamour, UMR CNRS 7198, Université de Lorraine, BP 70239, F 54506, Vandoeuvre lès Nancy, France
}

Marcel Hennes, Valentin Chardonnet, Emmanuelle Jal, Jan Lüning, and Boris Vodungbo

Sorbonne Université, CNRS, Laboratoire de Chimie Physique-Matière et Rayonnement, LCPMR, 75005, Paris, France

(Received 19 March 2019; revised manuscript received 16 September 2019; published 15 October 2019)

\begin{abstract}
During the past few years high-order harmonic generation (HHG) has opened up the field of ultrafast spectroscopy to an ever larger community by providing a table-top and affordable femtosecond extreme ultraviolet (EUV) and soft-x-ray source. In particular, the field of femtomagnetism has largely benefited from the development of these sources. However, the use of x-ray magnetic circular dichroism (XMCD) as a probe of magnetization, the most versatile and reliable one, has been constrained by the lack of polarization control at HHG sources, so studies have relied on more specific magneto-optical effects. Even the recent developments on the generation of elliptically polarized harmonics have only resulted in a few time-resolved experiments relying on this powerful technique since they add complexity to already-difficult measurements. In this article we show how to easily probe magnetization dynamics with linearly polarized EUV or soft-x-ray light with a versatility similar to XMCD by exploiting the Faraday effect. Static and time-resolved measurements of the Faraday effect are presented around the Co $M$ edges. Using simple theoretical considerations, we show how to retrieve the samples magnetization dynamics from the Faraday rotation and ellipticity transients. Ultrafast demagnetization dynamics of a few nanometers in Co-based samples are measured with this method in out-of-plane as well as in-plane magnetization configurations, showing its great potential for the study of femtomagnetism.
\end{abstract}

DOI: 10.1103/PhysRevB.100.144421

\section{INTRODUCTION}

High-order harmonic generation (HHG) is a table-top source of femtosecond coherent light covering the extreme ultraviolet (EUV) and soft-x-ray spectral ranges. Up to recent times, only linearly polarized HHG-based experiments were conducted. Indeed, although it has been known for more than 20 years that the harmonic beam somewhat retains the polarization state of the driving laser [1,2], nonlinearly polarized driving fields lead to a very low efficiency of elliptically polarized harmonics, preventing them from being used as a light source for experiments. To overcome this limitation, recent research on HHG has focused on the development of full polarization control of intense harmonic beams [3-12]. To produce high-intensity elliptically polarized harmonics for applications, several schemes have been proposed and implemented in the past 10 years, such as (i) the harmonic generation with aligned molecules [3,4,9], (ii) the use of a circular polarizer $[5,8]$, and (iii) harmonic generation with bicolor driving fields $[6,7,10]$. One of the strongest driving motivations behind the development of elliptically polarized

\footnotetext{
*guillaume.lambert@ensta-paristech.fr
}

HHG sources has been their application to the study of ultrafast magnetization dynamics $[5,7,8,10,13]$. Indeed, HHG sources are particularly suited for this type of study since they allow for nanometer-scale spectroscopic pump-probe experiments with unmatched time resolution-thanks to the very short harmonic pulse duration (down to the attosecond range) and its inherent synchronization with the driving laser (part of which is used as pump). In addition, thanks to harmonic polychromaticity and a photon energy range reaching the absorption edges of magnetic elements, simultaneous probing of different elements in alloy or heterostructures can be performed.

Linearly polarized harmonics can be used to probe magnetization by using resonant magneto-optical (MO) effects, such as the magneto-optical Kerr effect (MOKE) [14,15], with transverse (T-MOKE) being the preferred geometry with HHG, or resonant magnetic scattering [16,17]. These MO effects have been successfully exploited at HHG or other XUV sources during the past 10 years to study new aspects of ultrafast magnetization dynamics, such as element-specific or nanometer-scale dynamics [18-23]. However, the method of choice to probe magnetization in resonance with absorption edges is X-ray magnetic circular dichroism (XMCD). Indeed, contrary to T-MOKE or resonant magnetic scattering [24,25], 
the relationship between XMCD and magnetization is fairly linear and well established [26,27]. Moreover, it is a more versatile method since it can be used to probe uniformly magnetized samples in transmission and hence is not limited to samples presenting nanoscale magnetic domains, such as resonant magnetic scattering, and is not limited to probing the sample surface, such as T-MOKE.

As indicated by its name, time-resolved XMCD experiments require circularly, or at least elliptically, EUV or soft$\mathrm{x}$-ray photons. Despite the tremendous recent development of elliptically polarized HHG sources described earlier, there have been very few HHG-based time-resolved experiments relying on XMCD as a probe [8]. This is probably due to the fact that the schemes used to produce elliptically polarized harmonics require extra beams and optics compared with the classical technique. Consequently, they add complexity to the generation setup, reduce the harmonic yield, and introduce instability. All of these points are detrimental to the realization of sensitive time-resolved experiments.

In this report, we demonstrate how we can retain both the simplicity of a linearly polarized HHG source and the versatility of XMCD by using the Faraday effect as a resonant MO probe of magnetization for the study of ultrafast magnetization dynamics. Using cobalt-based samples, the static resonant Faraday effect have been measured at the $M_{2,3}$ edge of Co with our HHG source and, in particular, with an optimized polarizer this effect can be stronger than XMCD. Moreover, a time-resolved experiment has been implemented based on this effect in perpendicular (out-of-plane) and parallel (in-plane) magnetization geometries. We show that time-resolved signals can be obtained for samples as thin as a few nanometers magnetized in-plane. Finally, theoretical considerations allow us to retrieve simultaneously the magnetization dynamics from the Faraday effect transients for different harmonics, allowing us to obtain a complete spectroscopic picture of the magnetization dynamics.

\section{THEORETICAL CONSIDERATIONS}

Macroscopically, the Faraday effect modifies the polarization of a linearly polarized beam traveling through a magnetized medium, as presented in the inset of Fig. 1, as long as the magnetization axis is not perpendicular to the beam path. In the case of a nontransparent medium, the initially linearly polarized harmonics will become elliptically polarized (see Fig. 1 for the definition of Faraday ellipticity $\varepsilon_{F}$ ) with a polarization axis rotated compared with the original polarization by an angle $\theta_{F}$ (see Fig. 1).

If both the magnetization and the incident light are normal to the material's surface (i.e., parallel to each other), the incident linearly polarized light can be decomposed as the sum of two circularly polarized waves of positive and negative helicity with equal amplitude. The complex refractive indexes $n_{ \pm}$for these two circularly polarized waves describe the propagation of the radiation through the sample, where \pm refer to parallel and antiparallel orientation of the waves helicity relatively to the magnetization of the sample $(\overrightarrow{\mathrm{M}}) . n_{+}$and $n_{-}$ are defined as follows:

$$
n_{ \pm}=1-\left(\delta_{0} \pm \Delta \delta\right)+i\left(\beta_{0} \pm \Delta \beta\right) .
$$

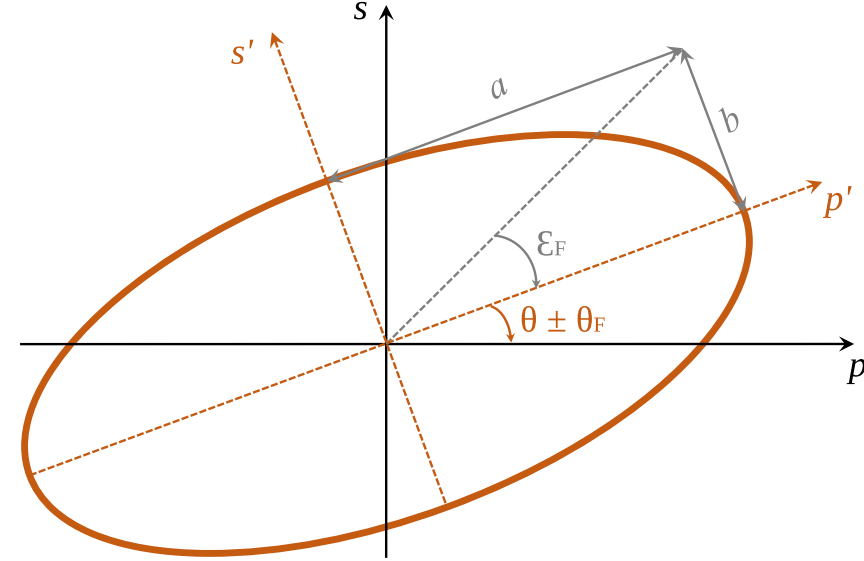

FIG. 1. The Faraday effect leads to a transmitted beam elliptically polarized with a rotation of the polarization $\theta_{F}$ and an ellipticity $\varepsilon_{F}$. The ellipse major and minor axes, $a$ and $b$, define the ellipticity as $\tan \varepsilon_{F}=b / a$. The $p^{\prime}$ and $s^{\prime}$ axes are rotated axes parallel to $a$ and $b$. The electric field of the beam is easily described in this coordinate system.

The real part corresponds to a phase shift of the radiation, while the imaginary part describes the absorption. $\Delta \delta$ and $\Delta \beta$ can be expanded as a series of powers of the magnetization $M$ but are largely dominated by the linear term. If one of the circular waves travels faster than the other through the material, constituting the phase shift, it will also rotate faster, generating the rotation of the light's polarization axis: this constitutes the Faraday rotation $\theta_{F}$, which is proportional to $\Delta \delta$ [28]. The difference in absorption of the two circularly polarized waves changes the polarization of the light from linear to elliptical: this constitutes the Faraday ellipticity $\varepsilon_{F}$, which is proportional to $\Delta \beta$ [28].

The principle of our Faraday effect measurement is presented in Fig. 2. The incident light polarization is described in the usual coordinate system, $p$ and $s$, where the $p$ axis is parallel to the plane of incidence and the $s$ axis is perpendicular, both being perpendicular to the beam path. If the beam incident on the sample is purely $p$ polarized, the Faraday effect will induce a modification of its polarization state resulting in the appearance of an $s$ component. Measuring this component allows us to quantify the intensity of the Faraday effect and the magnetization of the sample, as shown below. To do that, our method is based on a polarization analyzer, which ideally would completely suppress the $p$ component.

Let us now consider cases for which the measured effects will be dominated by the Faraday rotation (this is the case for most photon energies). For two opposite directions of the sample's magnetization $\left(\mathrm{M}^{-}\right.$and $\left.\mathrm{M}^{+}\right)$these $s$ components are of the same magnitude but of opposite sign [opposite rotation; see Fig. 2(a)]. Since the detector placed after the analyzer is only sensitive to the intensity of the beam-proportional to the square of the electric field-our single-analyzer setup, sensitive mainly to the $s$ component, cannot differentiate between these two opposite-magnetization states: they will yield the same intensity on the detector.

To distinguish between opposite magnetization directions, one has to rotate the incident polarization axis by a small 


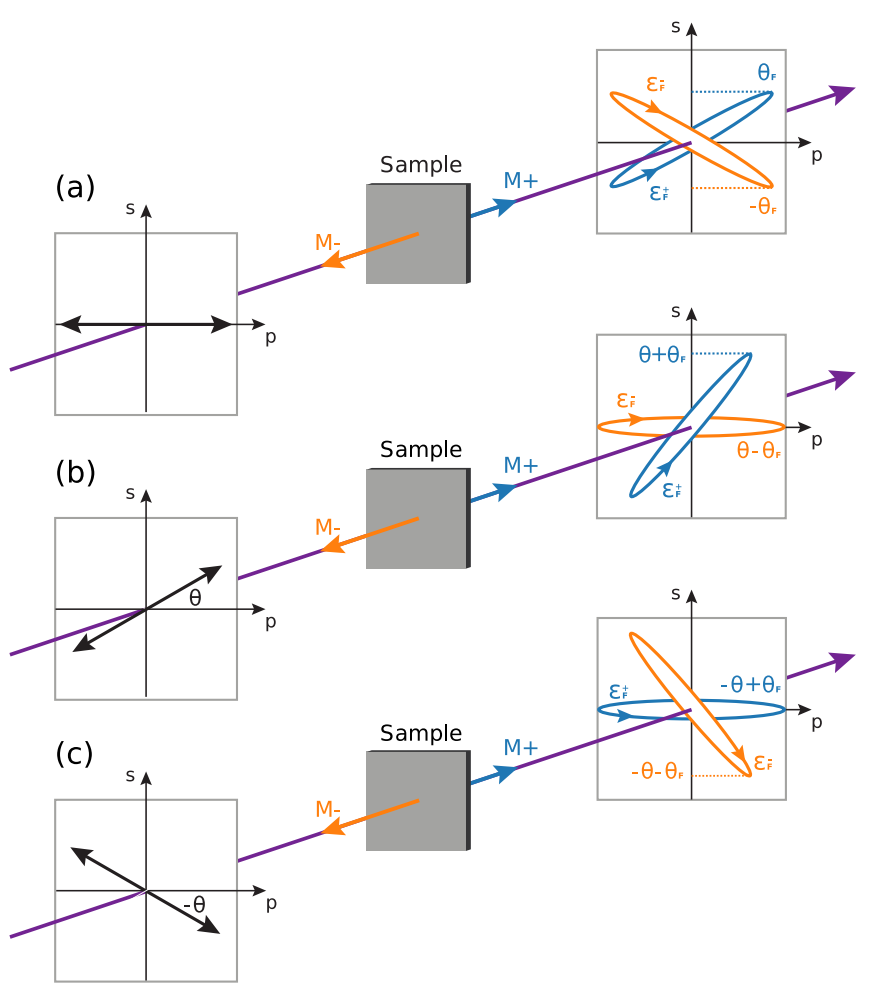

FIG. 2. Polarization of the harmonic beam after a magnetized sample for two opposite magnetization directions, $M^{+}$(blue) and $M^{-}$ (orange), starting from three different configurations of the incident polarization: (a) linearly $p$ polarized, (b) linearly polarized with an angle $\theta$ compared with the $p$ axis, and (c) linearly polarized with an angle $-\theta$ compared with the $p$ axis. Because of the Faraday rotation $\theta_{F}$ and ellipticity $\varepsilon_{F}$, the $s$ component of the electric field strongly depends on the sample's magnetization. For a $p$-polarized incident polarization, the $s$ component of the transmitted electric field is opposite for opposite magnetization, leading to the same measured intensity. A small rotation of the incident polarization $\theta$ allows us to differentiate between these two magnetization directions.

angle $\theta$. If $\theta \geqslant \theta_{F}$, the projection of the electric field along the $s$ axis after the sample increases for one magnetization direction and decreases for the other [Fig. 2(b)]. The signal measured after the analyzer then monotonically depends on the magnetization. It has to be noted that using an opposite polarization angle $-\theta$ reverses the signal [Fig. 2(c)]. The stronger the Faraday effect, the higher the difference between the signals measured for opposite magnetization directions. To maximize the ratio between the two different $s$ components of the electric field obtained upon reversal of the magnetization, one has to use a $\theta$ angle of the order of $\theta_{F}$. Indeed, if the light source is not perfectly stable, it can be better to maximize the ratio rather than the difference to obtain a very good contrast and a higher detection efficiency.

A more detailed analysis can be done by writing the electric field of one harmonic after the sample for the two opposite magnetization directions $\left(M^{+}\right.$and $\left.M^{-}\right)$, perpendicular to the sample's surface. As shown in Fig. 1, in the $\left(\vec{e}_{p}{ }^{\prime}, \vec{e}_{s}{ }^{\prime}\right)$ basis, the electric field can be simply written as follows:

$$
\vec{E}_{ \pm}=a \vec{e}_{p}^{\prime} \pm i b \vec{e}_{s}^{\prime}
$$

where $a$ and $b$ are respectively the major and minor axis of the polarization ellipse. To evaluate the effect of the analyzer on the beam, this equation has to be rewritten in the $\left(\vec{e}_{p}, \vec{e}_{s}\right)$ basis. This is done by writing $\vec{e}_{p}{ }^{\prime}$ and $\vec{e}_{s}{ }^{\prime}$ in the $\left(\vec{e}_{p}, \vec{e}_{s}\right)$ basis:

$$
\begin{aligned}
& \vec{e}_{p}{ }^{\prime}=\cos \left(\theta \pm \theta_{F}\right) \vec{e}_{p}+\sin \left(\theta \pm \theta_{F}\right) \vec{e}_{s}, \\
& \vec{e}_{s}{ }^{\prime}=-\sin \left(\theta \pm \theta_{F}\right) \vec{e}_{p}+\cos \left(\theta \pm \theta_{F}\right) \vec{e}_{s},
\end{aligned}
$$

where $\theta$ is the initial angle between the polarization axis and the $p$ direction (before the sample) and $\theta_{F}$ is the Faraday rotation. This allows us to rewrite Eq. (2) as follows:

$$
\begin{aligned}
\vec{E}_{ \pm}= & {\left[a \cos \left(\theta \pm \theta_{F}\right) \mp i b \sin \left(\theta \pm \theta_{F}\right)\right] \vec{e}_{p} } \\
& +\left[a \sin \left(\theta \pm \theta_{F}\right) \pm i b \cos \left(\theta \pm \theta_{F}\right)\right] \vec{e}_{s} .
\end{aligned}
$$

The analyzer has transmission coefficients $t_{p}$ and $t_{s}$ for the $p$ and $s$ directions, respectively, hence the electric field after the analyzer becomes

$$
\vec{E}_{ \pm}=E_{p}^{ \pm} \vec{e}_{p}+E_{s}^{ \pm} \vec{e}_{s}
$$

Since $\tan \varepsilon_{F}=b / a, E_{p}^{ \pm}$and $E_{s}^{ \pm}$are therefore equal to

$$
\begin{aligned}
& E_{p}^{ \pm}=a t_{p}\left[\cos \left(\theta \pm \theta_{F}\right) \mp i \tan \left(\varepsilon_{F}\right) \sin \left(\theta \pm \theta_{F}\right)\right], \\
& E_{s}^{ \pm}=a t_{s}\left[\sin \left(\theta \pm \theta_{F}\right) \pm i \tan \left(\varepsilon_{F}\right) \cos \left(\theta \pm \theta_{F}\right)\right]
\end{aligned}
$$

The total intensity of the transmitted light is

$$
I^{ \pm}=I_{p}^{ \pm}+I_{s}^{ \pm} \propto\left|E_{p}^{ \pm}\right|^{2}+\left|E_{s}^{ \pm}\right|^{2} .
$$

From Eq. (6), the $p$ and $s$ components of the intensity, $I_{p}^{ \pm}$and $I_{s}^{ \pm}$, respectively, can be written as follows:

$$
\begin{aligned}
& I_{p}^{ \pm} \propto a^{2} T_{p}\left[\cos ^{2}\left(\theta \pm \theta_{F}\right)+\tan ^{2}\left(\varepsilon_{F}\right) \sin ^{2}\left(\theta \pm \theta_{F}\right)\right], \\
& I_{s}^{ \pm} \propto a^{2} T_{S}\left[\sin ^{2}\left(\theta \pm \theta_{F}\right)+\tan ^{2}\left(\varepsilon_{F}\right) \cos ^{2}\left(\theta \pm \theta_{F}\right)\right],
\end{aligned}
$$

where $T_{p}=\left|t_{p}\right|^{2}$ and $T_{s}=\left|t_{s}\right|^{2}$. Since $\theta \pm \theta_{F} \ll 1$ and $\varepsilon_{F} \ll 1$ [29], Eq. (8) can be simplified to

$$
\begin{aligned}
& I_{p}^{ \pm} \propto a^{2} T_{p}\left[1+\varepsilon_{F}^{2}\left(\theta \pm \theta_{F}\right)^{2}\right] \sim a^{2} T_{p}, \\
& \left.I_{s}^{ \pm} \propto a^{2} T_{s}\left[\left(\theta \pm \theta_{F}\right)^{2}\right)+\varepsilon_{F}^{2}\right],
\end{aligned}
$$

which gives

$$
I^{ \pm} \propto a^{2}\left[T_{p}+T_{s}\left(\theta^{2} \pm 2 \theta \theta_{F}+\theta_{F}^{2}+\varepsilon_{F}^{2}\right)\right] .
$$

If we consider the difference between the harmonic intensity of the two opposite magnetization directions, we obtain

$$
I^{+}-I^{-} \propto a^{2} T_{s} 4 \theta \theta_{F} .
$$

At this stage, we have to consider that only $a$ and $\theta_{F}$ depend on the magnetization. Neglecting multiple internal reflections of the sample (which is most of the time justified for EUV and soft-x-ray radiation), it can be shown that $a$ is proportional to $E_{0} \cosh (k d \Delta \beta)$, where $E_{0}$ is the polarization of the incoming beam, $k$ is the wave vector, and $d$ is the thickness of the sample. For small values of $k d \Delta \beta$ (which is the case for us here), $\cosh (k d \Delta \beta)$ reduces to 1 and the parameter $a$ is mostly independent of the magnetization. Therefore we can write

$$
I^{+}-I^{-} \propto \theta_{F} \propto \Delta \delta \propto M .
$$

Consequently, the magnetization dynamics is proportional to the time evolution of the difference between $I^{+}$and $I^{-}$and one has only to measure the intensity of the transmitted light for the two opposite magnetization directions to retrieve it. 
On the other hand, for specific photon energies very close to the $M$ and $L$ absorption edges of magnetic transition metals, the Faraday rotation vanishes $\left(\theta_{F} \sim 0\right)$ and the signal is dominated by the Faraday ellipticity [29]. In that case, it becomes impossible to retrieve the magnetization by the previous method. However, the transmitted intensities reduce to

$$
I^{+}=I^{-} \propto a^{2}\left[T_{p}+T_{s}\left(\theta^{2}+\varepsilon_{F}^{2}\right)\right] .
$$

We can then perform an additional measurement for an unmagnetized sample $(M=0)$ for which the Faraday ellipticity is equal to zero, yielding an intensity $I(M=0) \propto a^{2}\left[T_{p}+\right.$ $\left.T_{s} \theta^{2}\right]$. The difference between the intensities measured for magnetized, $I(M)=I^{+}$or $I^{-}$, and unmagnetized, $I(M=0)$, samples is the given by

$$
I(M)-I(M=0) \propto a^{2} T_{s} \varepsilon_{F}^{2} \propto(\Delta \beta)^{2} \propto M^{2} .
$$

This difference is proportional to the square of the Faraday ellipticity $\varepsilon_{F}$, hence to the square of $\Delta \beta$ and finally to the square of the magnetization, $M^{2}$. Once again, one can easily retrieve the magnetization dynamic by measuring the time evolution of this difference. The zero-magnetization measurement has to be performed only once because it does not depend on the time delay.

It has to be noted that this analysis remains essentially valid for non-normal incidence. At the $L$ edges of transition metals, the optical index is very close to 1 and the proportionality between $\theta_{F}$ and $\Delta \delta$ and between $\varepsilon_{F}$ and $\Delta \beta$ holds [28]. At the $M$ edges, only a weaker approximation can be made but $\theta_{F}$ and $\varepsilon_{F}$ remain linearly proportional to a combination of $\Delta \delta$ and $\Delta \beta$ [29]. Consequently, $\theta_{F}$ and $\varepsilon_{F}$ are still proportional to the magnetization.

To verify the validity of Eqs. (12) and (14), we have perform simulations of the interaction between EUV light around the Co $M$ edge (close to $60 \mathrm{eV}$ ) and a Co/Pt multilayer using a matrix formalism [30] and magneto-optical constants recently measured [31]. In particular, the transmitted electric field and polarization have been calculated in the configuration described above, i.e., a linearly polarized incident wave slightly rotated compared with the $p$ axis impinging on the sample at normal incidence. Those simulations confirm that there is a linear relationship between $I^{+}-I^{-}$and the sample magnetization for photon energies where the Faraday rotation can be measured. They also confirm that Eq. (14) can be used in a photon energy range of about $1 \mathrm{eV}$ around the energy where the Faraday rotation vanishes.

\section{EXPERIMENTAL DETAILS}

Our experimental proof of principle is carried out by using a $5 \mathrm{kHz}$ laser system at $\sim 800 \mathrm{~nm}$ with a pulse duration of about $30 \mathrm{fs}$ and $2 \mathrm{~mJ}$ per pulse, which is split into pump and probe beams by a beam splitter. Figure 3 represents schematically the experimental setup.

The linearly polarized laser is focused into a gas cell filled with neon to generate high-order harmonics by nonlinear interaction between the laser and the gas. The generated harmonics maintain the polarization properties of the infrared laser and span a wide photon energy range. A half-wave plate is used to control the polarization axis of the laser and hence the linearly polarized harmonics. By adjusting the source parameters, such as the iris aperture, the laser chirp, and the gas pressure, it is possible to optimize the generation efficiency close to the targeted spectral range for magnetic spectroscopy studies. Three $\mathrm{SiO}_{2}$ mirrors at grazing incidence and a 300-nm-thick Al foil are used to remove the infrared (IR) radiation after the gas cell and a toroidal mirror focuses the harmonics onto the sample.

The magnetic field applied to the samples can be either out-of-plane or in-plane. For the first one, the sample is surrounded by four permanent magnets (Fig. 3). This magnet arrangement generates a field of $200 \mathrm{mT}$ perpendicular to the sample surface, which is sufficiently intense to fully saturate the magnetization of the samples. For the in-plane geometry, only two magnets are used and the field reaches $140 \mathrm{mT}$. For both configurations the magnet structure can be rotated around the sample to reverse the magnetization of the sample. Different magnetic samples were used in the experiment: a CoDy alloy film: $\mathrm{Ta}(3 \mathrm{~nm}) / \operatorname{Pt}(5 \mathrm{~nm}) / \mathrm{Co}_{76} \mathrm{Dy}_{24}(50 \mathrm{~nm}) / \operatorname{Pt}(5$ $\mathrm{nm})$, two Co/Pt multilayers CoPt-1: $\mathrm{Pt}(2 \mathrm{~nm}) /[\mathrm{Co}(0.6 \mathrm{~nm}) /$ $\mathrm{Pt}(0.8 \mathrm{~nm})] / \mathrm{Al}(5 \mathrm{~nm}) \times 20 / \mathrm{Al}(5 \mathrm{~nm})$ and CoPt-2: $\mathrm{Ta}(5 \mathrm{~nm}) /$ $\mathrm{Pt}(5 \mathrm{~nm}) /[\mathrm{Co}(1.5 \mathrm{~nm}) / \operatorname{Pt}(1.2 \mathrm{~nm})] \times 5 / \operatorname{Pt}(3 \mathrm{~nm})$, and a single Co layer $\mathrm{Ta}(5 \mathrm{~nm}) / \operatorname{Pt}(5 \mathrm{~nm}) / \operatorname{Co}(5.5 \mathrm{~nm}) / \operatorname{Pt}(3 \mathrm{~nm})$. All the samples were deposited by sputtering onto 50 -nm-thick $\mathrm{Si}_{3} \mathrm{~N}_{4}$ membranes with a surface of $0.05 \times 0.05 \mathrm{~mm}^{2}$.

After the sample, the harmonics are reflected by a polarization analyzer into a spectrometer consisting of a concave grating (1200 lines/mm) and a CCD camera. Using the polarization analyzer at the Brewster angle, the $p$ reflectivity $R_{p}$ is very weak and, consequently, the intensity recorded on the CCD is mostly due to the $s$ component of the electric field. To increase the sensitivity of our measurement we therefore have to maximize $R_{s}$ and $\frac{R_{s}}{R_{p}}$. This can be done by using a metallic mirror with fairly good reflectivity in the EUV spectral range, e.g., a gold mirror $\left(R_{s}=0.04\right.$ and $\frac{R_{s}}{R_{p}}=17$ for $60 \mathrm{eV}$ at $40^{\circ}$ ), or a broadband multilayer mirror optimized for the targeted spectral range between 50 and $70 \mathrm{eV}\left(R_{s}=\right.$ 0.18 and $\frac{R_{s}}{R_{p}}=900$ for $60 \mathrm{eV}$ at Brewster's angle). The goldcoated mirror and the multilayer mirror optimized for $60 \mathrm{eV}$ have their respective Brewster angles at $40^{\circ}$ and $42.1 .^{\circ}$ The first one was used to demonstrate the applicability of the method on the thick CoDy sample. The second one was used to measured smaller effects on the thinner $\mathrm{Co} / \mathrm{Pt}$ and $\mathrm{Co}$ samples.

For static experiments, we measured the harmonics intensity for two opposite magnetization directions of the sample and for two opposite polarization angles for a complete demonstration of the Faraday effect. To perform the timeresolved experiment, a small fraction of the infrared laser beam, which can be controlled in time delay compared with the main laser beam driving the HHG process, is used as a pump to initiate the demagnetization dynamics. Since pump and probe pulses originate from the same laser pulse, this setup is inherently jitter free, allowing us to follow the magnetization dynamics with a time resolution slightly higher than $30 \mathrm{fs}$, since the HHG pulse duration is much smaller than the laser pulse. 


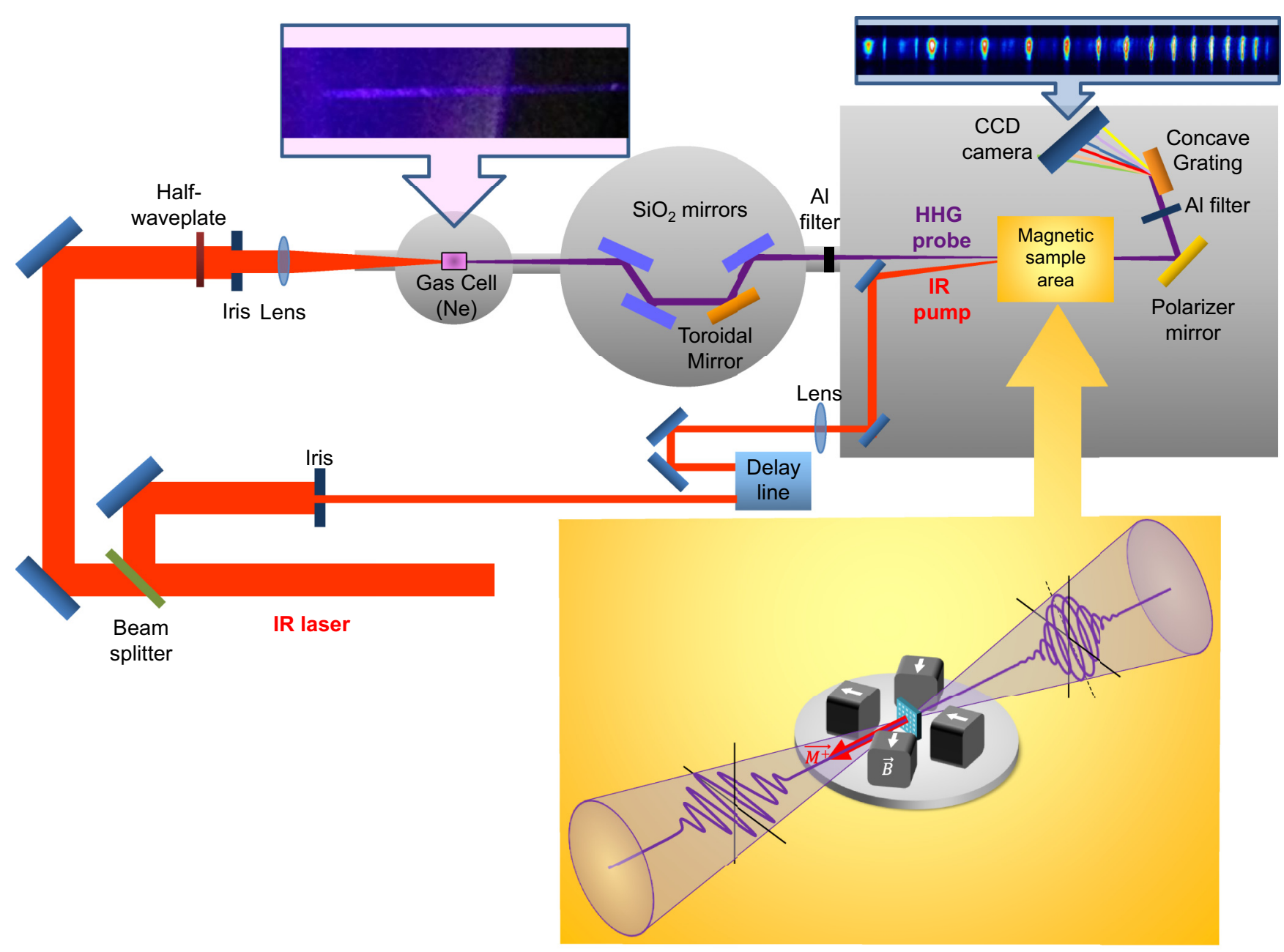

FIG. 3. Schematic diagram of the experimental setup. The magnetic sample is pumped by an IR laser pulse and probed by a delayed linearly polarized harmonic pulse, generated by the same laser in a gas cell filled with neon. Both pump and probe are focused at the same position onto the sample and are almost in collinear geometry perpendicular to its surface. The analyzer is set at the Brewster angle for $60 \mathrm{eV}$ radiation, i.e., reflecting mainly the $s$ component of the harmonic beam. The harmonics are then separated and detected by a spectrometer composed of a concave grating and a CCD camera. A set of three $\mathrm{SiO}_{2}$ mirrors and aluminum filters shield the detector from the IR light. (inset) The Co based samples are surrounded by a rotating assembly of four permanent magnets which can magnetize the sample at saturation out-of-plane in both directions.

\section{RESULTS AND DISCUSSION}

The transmission efficiency of the high-order harmonics around the spectral energy range of interest was evaluated for the CoDy sample. Figure 4(a) represents the spectral distribution of the high-order harmonics recorded by the CCD camera for the $M^{-}$direction. Figure 4(b) shows the projection of the intensity of two harmonics spectra recorded with (magenta) and without (black) the CoDy sample. When the sample is in the harmonics path, the transmission efficiency of the harmonics around the $\mathbf{M}_{2,3}$ absorption edge of cobalt is calculated. As expected, there is a strong absorption of the signal around this edge. The transmission decays from $10 \times 10^{-3}$ at $57.7 \mathrm{eV}$ to $1.5 \times 10^{-3}$ at $60.8 \mathrm{eV}$ [Fig. 4(b), right axis].

For the same CoDy sample, Fig. 5(a) shows the projection of the energy of the harmonics spectra for the two opposite magnetization directions $\left(M^{-}\right.$in blue and $M^{+}$in red). The curves correspond to an average of the harmonics spectra recorded for each magnetization direction. For nonzero $\theta$ we observe a different response in the intensity signal of the harmonics for the two magnetization directions: for $M^{-}$ the intensity of the harmonics is higher than for $M^{+}$. This observation is in agreement with our theoretical description of the experiment (vertical projection of the ellipsis illustrated in Fig. 2). The Faraday effect is particularly pronounced for harmonics from 54 to $60 \mathrm{eV}$, consistent with previous works [29].

To quantify the static Faraday effect for each harmonic, the asymmetry $A$, defined as

$$
A=\frac{I^{+}-I^{-}}{I^{+}+I^{-}},
$$

has been calculated thanks to the two magnetization directions $\left(M^{-}\right.$and $\left.M^{+}\right)$. Figure 5(b) shows the results for the three different incident polarization axis $(\theta,-\theta$, and 0$)$. When the incident polarization is parallel to the analyzer plane $\left[\theta=0^{\circ}\right.$, as represented in Fig. 2(a)] the intensities recorded 


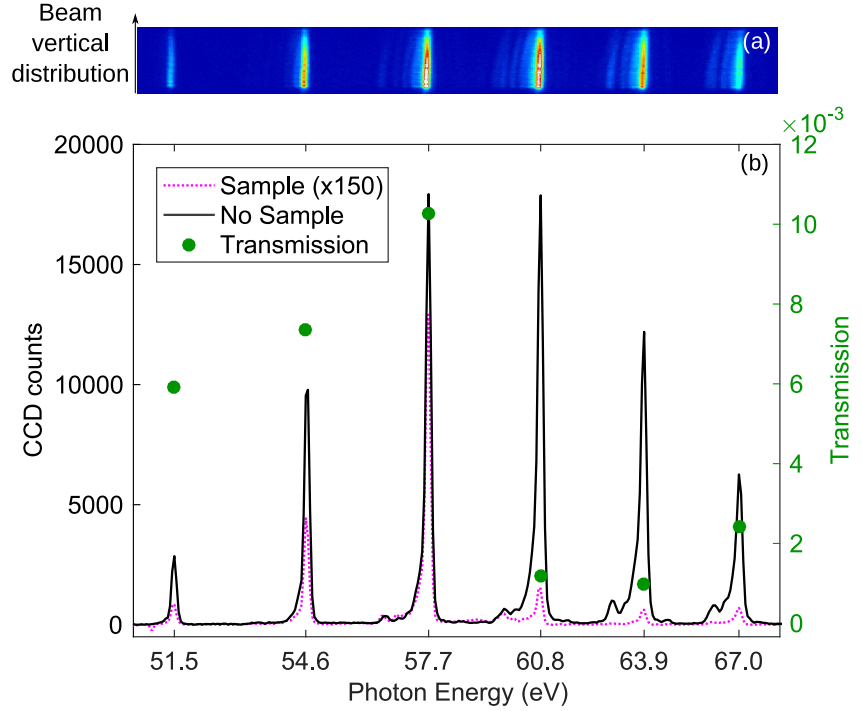

FIG. 4. (a) Harmonics spectrum: vertical distribution of the beam as function of the photon energy (30 s acquisition time). (b) Energy projection of the harmonics spectra showing the intensity of each harmonic for two configurations, with and without the sample in the harmonics path (30 and $2 \mathrm{~s}$ acquisition time, respectively).

for opposite magnetization have the same amplitude, and, as expected, $A \approx 0$.

To maximize the asymmetry, we rotated the incident polarization axis with respect to the $p$ direction by an angle $\theta$ to minimize the $s$ component of the electric field of one

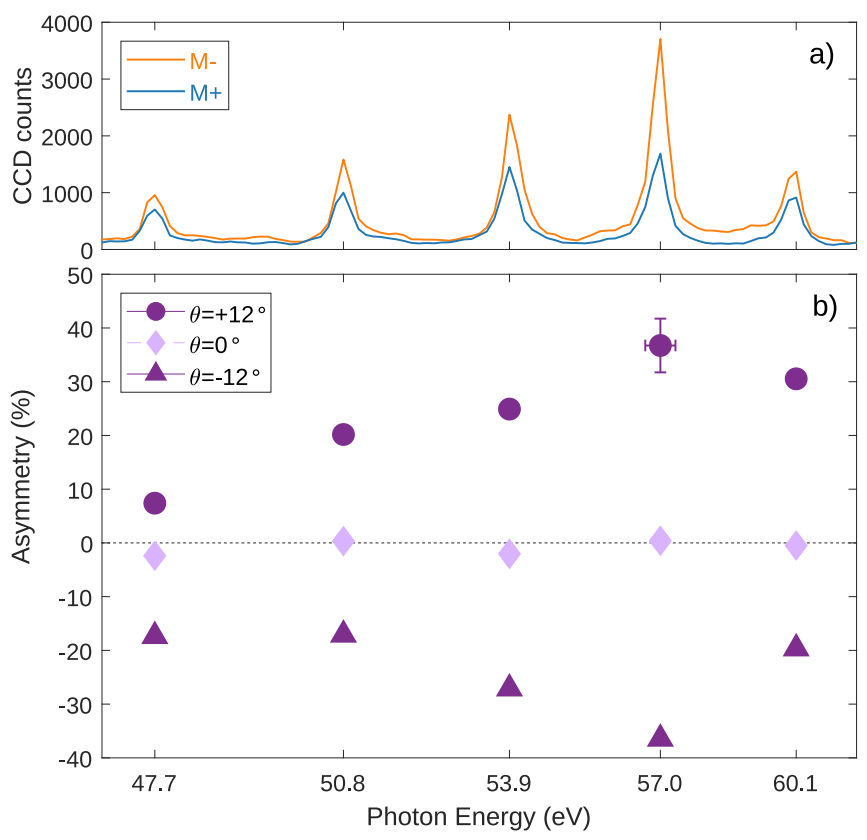

FIG. 5. Static Faraday effect of the CoDy sample as a function of photon energy in out-of-plane geometry. (a) Average of the energy projection of the recorded harmonics spectra showing the intensity of each harmonic for two opposite directions of the sample's magnetization $\left(M^{-}\right.$in orange and $M^{+}$in blue lines). (b) Asymmetry of the Faraday effect [see Eq. (15)] measured for three different polarization axis angles of the incident light $(\theta,-\theta$, and 0$)$.

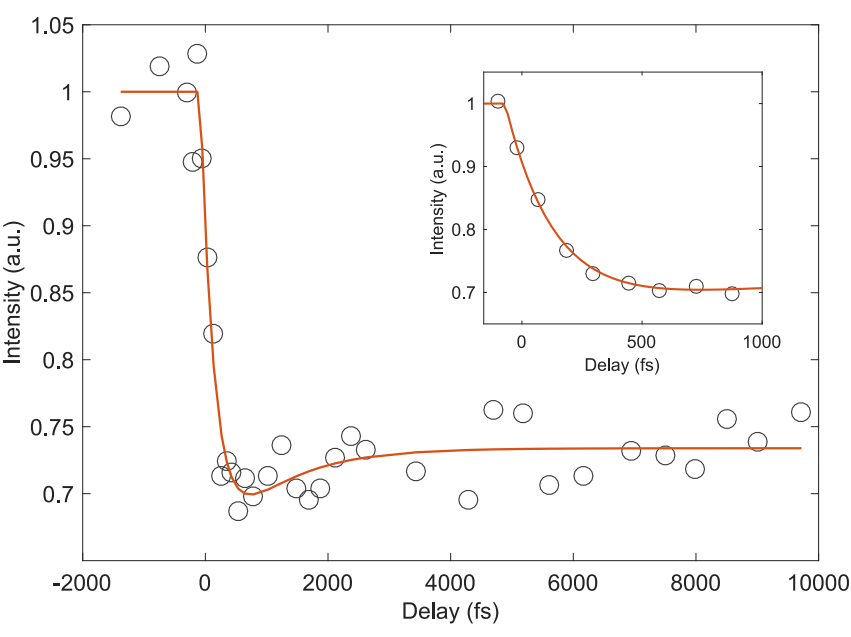

FIG. 6. Harmonic intensity as a function of time delay obtained from the CoDy sample magnetized out-of-plane $\left(M^{-}\right)$for a pump fluence of $5 \mathrm{~mJ} \mathrm{~cm}^{-2}$ and at a photon energy of $57 \mathrm{eV}$. The points have been obtained by an average over three acquisitions of $20 \mathrm{~s}$. The inset shows data from another scan focused on the short delays. The orange solid lines in both curves represent the best fits obtained.

of the two magnetization directions [Fig. 2(b)]. Using the opposite angle reverses the asymmetry [Fig. 2(c)]. For a gold analyzer around the Co $M$ edge the best angles that optimize the contrast between opposite magnetizations were found to be close to $\theta= \pm 12^{\circ}$, for which $A$ reaches almost $40 \%$ at $57 \mathrm{eV}$. Even with this not-so-small value for theta, our main approximation $\sin \left(\theta+\theta_{F}\right) \approx \theta+\theta_{F}$ is mostly valid since, in the worst-case scenario, $\theta=\theta_{F}=12^{\circ}=0.42 \mathrm{rad}$ and $\sin (0.42) \approx 0.41$. Figure 5 (b) clearly shows that the asymmetry can be measured over a photon energy range of at least $10 \mathrm{eV}$ (between 50 and $60 \mathrm{eV}$ ). This result demonstrates our ability to measure the Faraday effect in resonance. We note that the measured asymmetry is almost three times higher than what could be achieved on a sample of similar thickness using XMCD techniques. Indeed, Willems et al. [8] observed $A \approx 6 \%$ at the Co $M$ edge for a 15 -nm-thick sample which would yield $A \approx 15 \%$ for $38 \mathrm{~nm}$ of Co (the equivalent Co thickness of our CoDy sample). Finally, the high sensitivity of this effect allows us to measure effects far from the absorption edge. This broad spectral response could question the possibility to obtain element specificity in complex materials composed of different metals, especially here for $M$-edges resonances that are close to each other (iron, cobalt, nickel, etc.). In reality, close to the absorption edge of one element where the magneto-optical response is maximum, contributions from other elements are rather weak [31]. In addition, it has to be noted that this issue would not be specific to the Faraday effect and would affect all resonant magneto-optical probes of magnetization (XMCD, T-MOKE, and resonant scattering) at the $M$ edges of transition metals.

Time-resolved measurements on the same sample are shown in Fig. 6. The IR pump triggers the demagnetization dynamics and the harmonics probe the CoDy sample magnetic state at different time delays. Here we show the signal intensity as a function of time delay recorded for a probe photon energy of about $57 \mathrm{eV}$ and for only one direction 


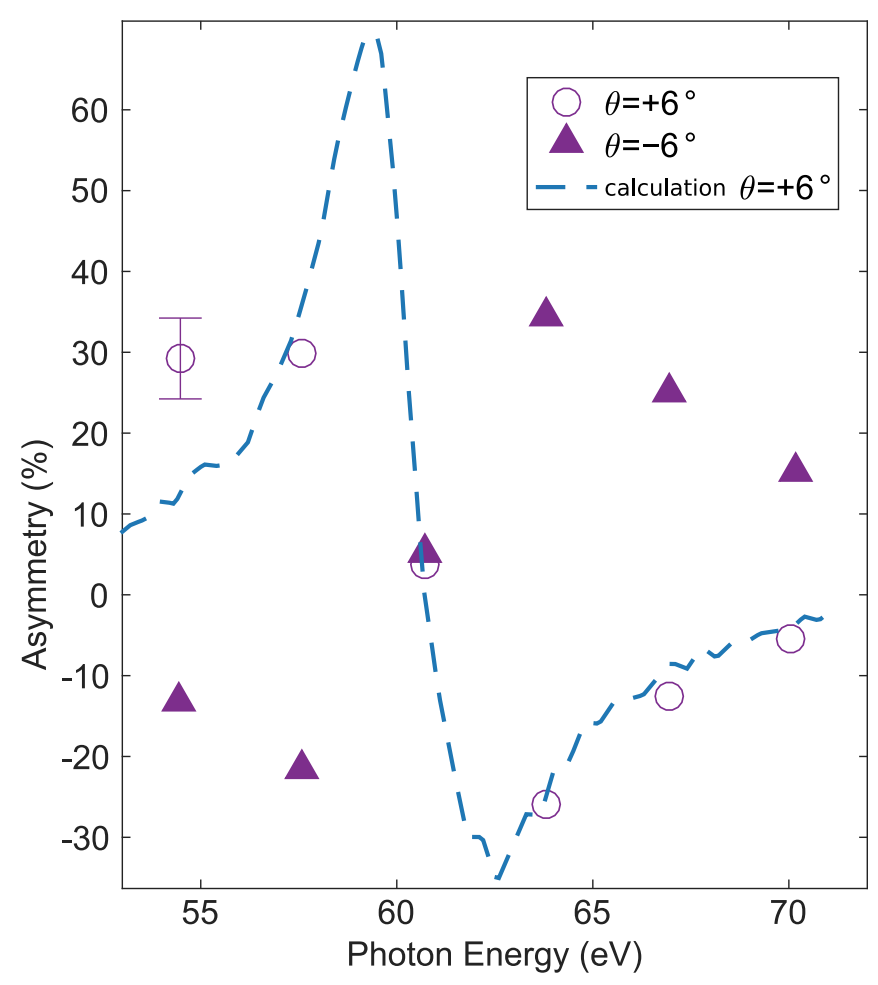

FIG. 7. Asymmetry as a function of photon energy for two opposite incident polarization axes $(\theta$ and $-\theta)$ recorded for the CoPt-1 sample showing a strong Faraday rotation below and above $60 \mathrm{eV}$ and a vanishing Faraday rotation close to $60.5 \mathrm{eV}$. The calculated asymmetry of a 12-nm-thick Co layer is shown (dashed blue line) for comparison and is in good agreement with the measurements.

of the magnetization. After excitation of the sample, the signal intensity rapidly decreases, staying flat up to $10 \mathrm{ps}$. As discussed in the theoretical part, for only one direction of the magnetization, the intensity recorded and shown on this curve is related to the magnetization but not directly proportional to it. We can nevertheless fit our data with a double exponential expression based on a semi-empirical model [32] to extract a rough estimate of the demagnetization time: in this case about $200 \mathrm{fs}$, in agreement with recent reports on magnetization dynamics in CoDy [33]. These first results prove the capability of our technique to follow magnetization dynamics.

To further enhance the sensitivity of our technique, we replaced the gold analyzer by a wide-band multilayer mirror optimized for the spectral range around $60 \mathrm{eV}$. This allowed us to study a $\mathrm{Co} / \mathrm{Pt}$ multilayer (CoPt-1) containing only 12 $\mathrm{nm}$ of Co (three times less than the CoDy sample). The static Faraday effect is shown in Fig. 7 for two opposite polarization axes of the incident light $\left(\theta= \pm 6^{\circ}\right.$, which maximizes the contrast between opposite magnetizations), and reaches about $30 \%$, which is a very high value for such a thin sample, now about six times higher than in XMCD [8]. Thanks to this multilayer mirror, having high reflectivity of the $s$ component and a higher ratio between $s$ and $p$ components, and a much smaller sample thickness, we were able to measure a clear Faraday signal even above $60 \mathrm{eV}$. This signal is reversed compared with the one below $60 \mathrm{eV}$. Using the magnetooptical constants recently measured by Willems $e t$ al. at the
$M$ edge of cobalt [31] and the measured reflectivity of the analyzer, we calculated the asymmetry of a 12 -nm-thick Co layer. We neglected multiple internal reflections and used only the transmission. The calculation and our results are in good agreement. The calculation also shows that we could get an even greater signal if we had a harmonic closer to $59 \mathrm{eV}$ where the maximum of the asymmetry is reached.

Time-resolved results obtained on a $\mathrm{Co} / \mathrm{Pt}$ multilayer are represented in Fig. 8. They were conducted on the CoPt-2 sample, which contains even less Co than the CoPt-1 sample (only $7.5 \mathrm{~nm}$ of $\mathrm{Co}$ ). Three different probe photon energies close to the Co $M$ edge are shown for two opposite magnetization directions of the sample $\left(M^{+}\right.$in blue and $M^{-}$in orange), corresponding to the configuration of Figs. 2(b) and 2(c). For harmonics at 57.4 and $63.6 \mathrm{eV}$, the time-dependent signals evolve in opposite direction for the two magnetization states [Figs. 8(a) and 8(c)]. From our theoretical analysis, this can be understood as follows: (i) the magnetization is reduced upon femtosecond excitation; (ii) the magnetizationdependent phase shift $\Delta \delta$ decreases proportionally; (iii) the Faraday rotation decreases and the polarization axis of the transmitted harmonics gets closer to its initial value at an angle $\theta$, from the $p$ direction [Figs. 8(d), 8(f), 8(g), and 8(i)]; (iv) the $s$ component of the harmonic electric field, which is preferentially reflected by the analyzer, tends to its value for an unmagnetized sample; and (v) the signal for the two magnetization states get closer (one increases the other decreases).

On the other hand, at $60.5 \mathrm{eV}$, the signal exhibits the same behavior for the two opposite magnetization directions. As observed on the static measurements, at this photon energy the Faraday rotation vanishes (Fig. 7). Consequently, the dynamic signal is the result of the time evolution of the Faraday ellipticity only and it is almost identical for the two opposite magnetization directions [Figs. 8(e) and 8(h)]. Indeed, as the magnetization decreases after excitation, the Faraday ellipticity reduces as well as the harmonic intensity after the polarizer.

To extract the magnetization dynamic from these signals, one has to apply Eqs. (12) and (14): when the Faraday rotation dominates the signal $(57.4$ and $63.6 \mathrm{eV})$, the magnetization dynamics is given by the difference between the intensities recorded for opposite magnetization, as shown in Fig. 9; when the Faraday ellipticity dominates $(60.5 \mathrm{eV})$, the magnetization dynamics is given by the square root of the difference between the intensity recorded for magnetized and unmagnetized (measured by magnetizing the sample perpendicularly to the probe beam) sample, as shown in Fig. 9.

We remark that the magnetization dynamics recorded at 57.4 and at $63.6 \mathrm{eV}$ (fit 1) are very similar but rather different from the one at $60.5 \mathrm{eV}$ (fit 2). The former is slower, $\tau_{M}=$ $125 \pm 10 \mathrm{fs}$, but stronger, $\frac{M_{\min }}{M_{0}}=0.36 \pm 5$ compared with the latter, where $\tau_{M}=90 \pm 10$ fs and $\frac{M_{\min }}{M_{0}}=0.45 \pm 5$. These two parameters, $\tau_{M}$ and $\frac{M_{\min }}{M_{0}}$, are respectively the delay for which the demagnetization reaches two-thirds-more exactly [1$\exp (-1)]$ - of its maximum value and the minimum value of the normalized magnetization. Because of the different shape of these two curves, one can safely exclude a normalization issue (with the zero-magnetization measurement, for example) 


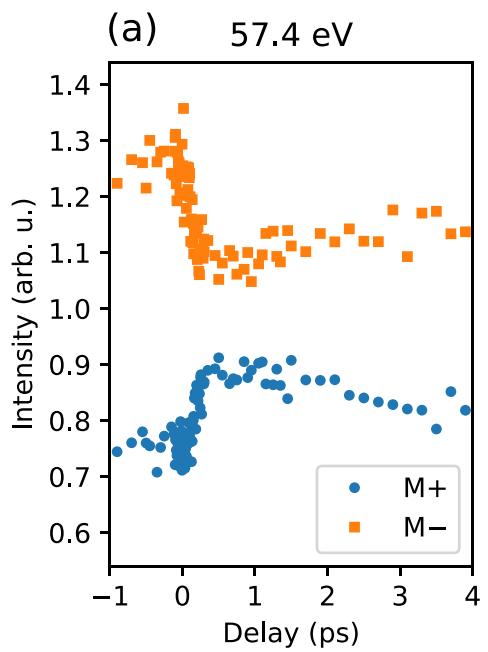

(b) $60.5 \mathrm{eV}$

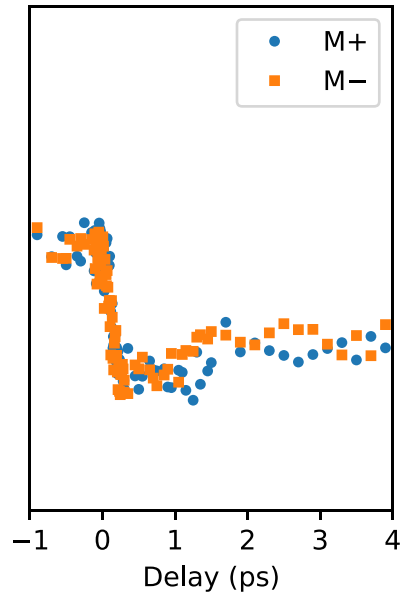

(e)

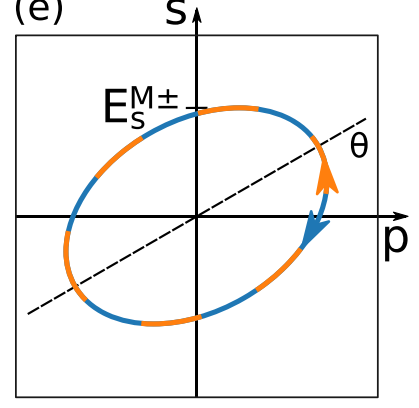

(h)

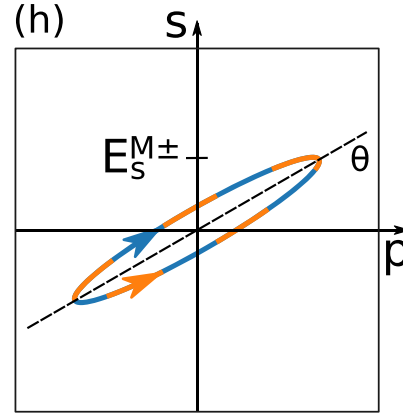

(c) $\quad 63.6 \mathrm{eV}$

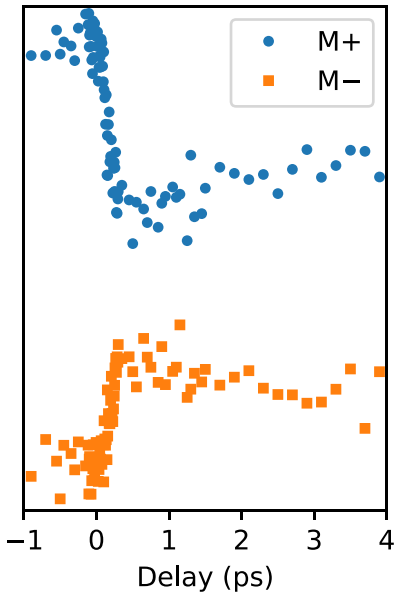

(f)

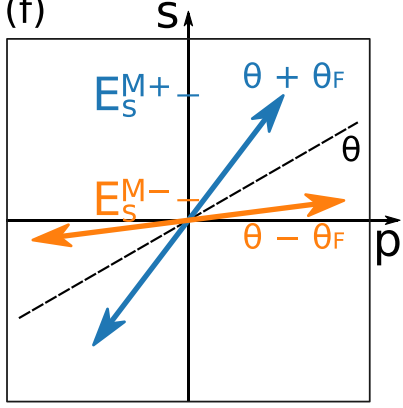

(i)

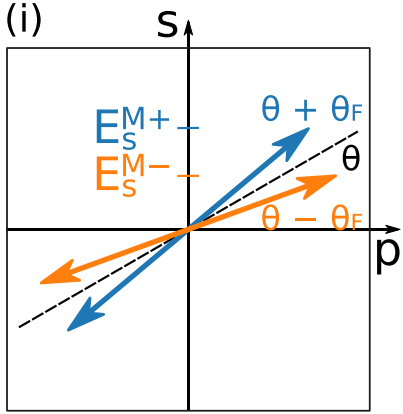

FIG. 8. Harmonic intensity as a function of time delay for three different photon energies: (a) $57.4 \mathrm{eV}$, (b) $60.5 \mathrm{eV}$, and (c) $63.6 \mathrm{eV}$ recorded for a $\mathrm{Co} / \mathrm{Pt}$ multilayer sample (Co-Pt2). For each energy, the Faraday effect was measured for two opposite magnetization directions of the sample $\left(M^{+}\right.$in blue and $M^{-}$in orange). Each point represents an acquisition of $20 \mathrm{~s}$. At 57.4 and $63.6 \mathrm{eV}$ the two curves start at different levels and get closer upon excitation by a femtosecond IR pulse. This can be understood in term of a reduced magnetization and hence Faraday rotation after the pump [panels (g) and (i)] compared with the unpumped state [panels (d) and (f)]. As expected from the static Faraday measurements (Fig. 7), the transient variations of the signal are reversed for 57.4 and $63.6 \mathrm{eV}$. As shown earlier, the Faraday rotation vanishes at $60.5 \mathrm{eV}$ (Fig. 7) and the two transients overlap. These two curves reveal a strong variation of the Faraday ellipticity, [panels (e) and (h)]. Since our setup is not sensitive to the helicity of the light, the response for opposite magnetizations is the same.

to explain these differences. A possible explanation would be that, in addition to the magnetization dynamics, we are sensitive to ultrafast modification of the electronic structure, which could shift the absorption edge, leading to photonenergy-dependent dynamics [34,35]. This point, which is a fundamental question of magneto-optic, cannot be resolved easily and requires us to be really cautious. However, the use of Faraday effect will help to conduct the much deeper investigations needed to address this question.

So far, we have reported results on samples magnetized out-of-plane. This geometry increases the Faraday signal but it requires samples with an out-of-plane magnetic anisotropy.
Unfortunately, magnetic thin films are most commonly easier to magnetize in-plane. For example, single layers of ferromagnetic transition metals ( $\mathrm{Fe}, \mathrm{Co}, \mathrm{Ni}$ ) with a thickness of few to tens of nanometers exhibit an in-plane magnetic anisotropy. We applied our method to such a Co single layer by slightly modifying the setup geometry: the sample is magnetized inplane but rotated by $30^{\circ}$ with respect to the incoming beam (see inset of Fig. 10). It has to be noted that, in this geometry, the Faraday rotation and ellipticity are reduced by about a factor of two due to the fact that we are only sensitive to the component of magnetization parallel to the beam (here only half of the total magnetization). 


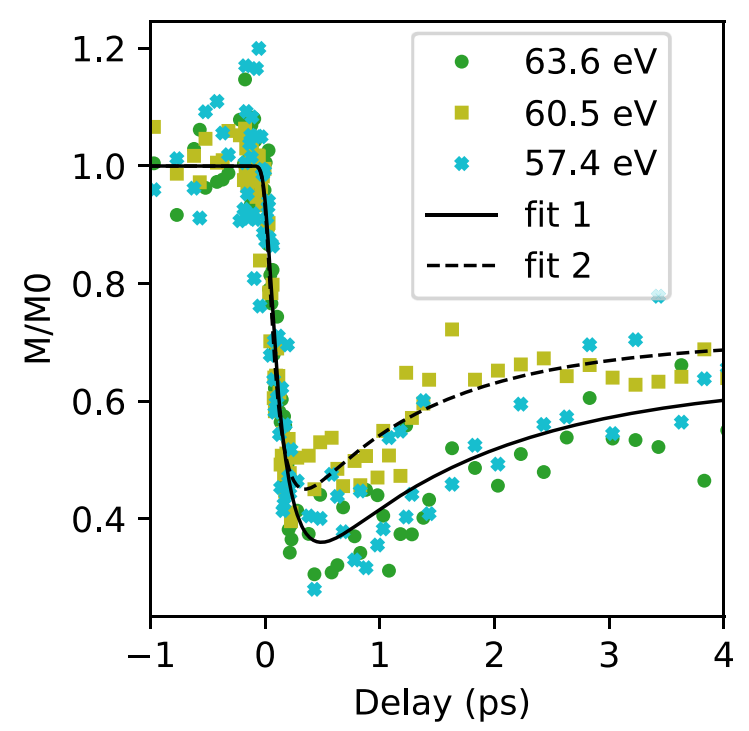

FIG. 9. Normalized magnetization as a function of time delay recorded with three different probe photon energies $(57.4,60.5$, and $63.6 \mathrm{eV}$ ) for the CoPt-2 sample magnetized out-of-plane. The curves are obtained from Fig. 8 and Eqs. (12) and (14). The value of $I(M=0)$ is the average obtained over a full delay scan. No features are visible around $t=0$ on this scan showing that $I(M=0)$ is constant. The pump fluence used was about $5 \mathrm{~mJ} \mathrm{~cm}^{-2}$. While the curves recorded at 57.4 and $63.6 \mathrm{eV}$ present similar evolutions, the curve recorded at $60.5 \mathrm{eV}$ presents a slightly faster demagnetization time and a reduced demagnetization rate, as best revealed by the fits obtained for 57.4 and $63.6 \mathrm{eV}$ (solid line) and for $60.5 \mathrm{eV}$ (dashed line).

The results obtained for this sample are reported in Fig. 10. Despite the unfavorable geometry we could record a demagnetization curve at the cobalt $M$ edge $(57.4 \mathrm{eV})$ for a sample consisting of only $7.5 \mathrm{~nm}$ of Co. This measurement demonstrates the very high sensitivity of our method. It also demonstrates that the method can be applied to a large variety of samples since most magnetic thin films exhibit an in-plane magnetic anisotropy. One can then observe on the demagnetization curves that the pure Co dynamics is quite a bit slower $\left(\tau_{M}=175 \pm 20 \mathrm{fs}\right)$ than the $\mathrm{Co} / \mathrm{Pt}$ multilayer dynamic $\left(\tau_{M}=125 \pm 10 \mathrm{fs}\right)$ for the same photon energy $(57.4 \mathrm{eV})$ and for a similar demagnetization $\left(\frac{M_{\min }}{M_{0}}=0.45 \pm\right.$ 10). These shorter demagnetization times in $\mathrm{Co} / \mathrm{Pt}$ multilayer compared with pure Co has already been observed with an all-optical pump-probe MOKE setup [36] but it would be very interesting to revisit this type of study in transmission with element specificity, i.e., with EUV or soft-x-ray photons, to determine the pure Co response and the importance of surface effects.

\section{CONCLUSION}

In conclusion, we show how one can measure femtosecond magnetization dynamics by using linearly polarized EUV or soft-x-ray sources in conjunction with the Faraday effect. This method was implemented in an HHG source setup and applied to the study of Co-based samples by probing Co magnetization with photons in resonance with the Co $M$ absorption

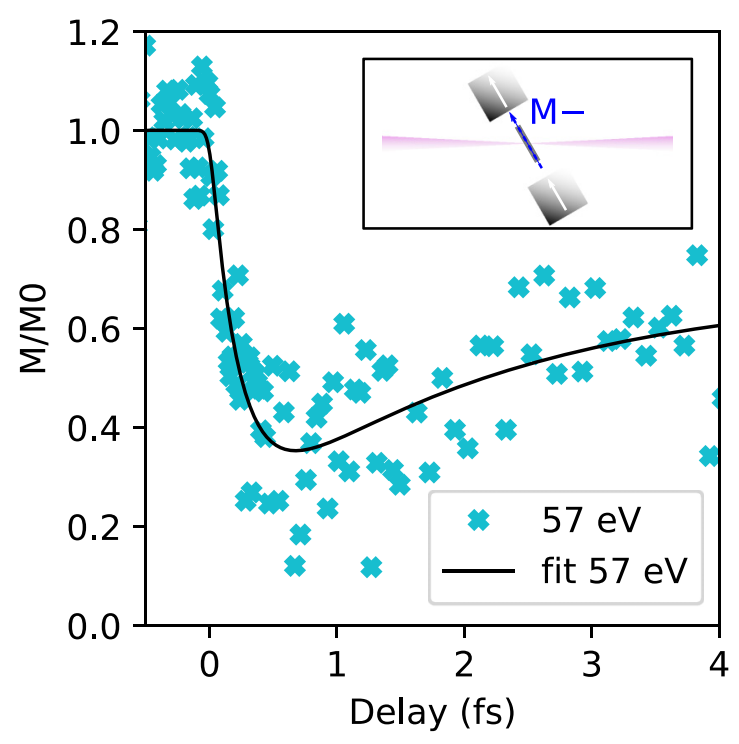

FIG. 10. Normalized magnetization as a function of time delay recorded with at $57.4 \mathrm{eV}$ for the Co sample magnetized in-plane. The solid line corresponds to the best fit. The inset represents the experimental magnetic geometry, in which the incidence angle on the sample is $30^{\circ}$. It has to be noted that, in this geometry, the apparent magnetization of the sample (the magnetization seen by the probe beam) is about half of the saturation magnetization, resulting in a lower Faraday signal. Nevertheless, the demagnetization is clearly visible, revealing a slower dynamic than the $\mathrm{Co} / \mathrm{Pt}$ multilayer at same probe energy. The pump fluence used was also $5 \mathrm{~mJ} \mathrm{~cm}^{-2}$.

edges. Ultrafast magnetization dynamics of a $\mathrm{Co} / \mathrm{Pt}$ multilayer film as well as a Co film containing both an equivalent of only $7.5 \mathrm{~nm}$ of Co have been measured respectively in out-ofplane and in-plane magnetization geometries, demonstrating the sensitivity and versatility of this method. Our report will undoubtedly open up the way to a new type of HHG-based studies in the field of femtomagnetism since our method does not require elliptical polarization (such as XMCD), does not require specific magnetic configuration of the sample (such as resonant magnetic scattering), and is not limited to the study of surface (such as T-MOKE). It can consequently be applied to the vast majority of magnetic thin-film systems.

Our technique is also well suited to take full advantage of the unique capabilities of free-electron lasers. In particular, the Faraday effect could be exploited to study ultrafast magnetization dynamics at the $L$ edges of transition metals and at the $M$ edges of rare-earth elements at photon energies not yet accessible for applications with HHG sources. Indeed, a thorough comparison between $M$ and $L$ absorption edges is needed to explain some discrepancies between previous reports in systems such as iron-nickel alloys [22,37]. Moreover, the Faraday effect could be used as magnetic probe in experiments, taking advantage of the very high number of photons per pulse at free-electron lasers, such as singleshot recording of magnetic dynamics [38]. Finally, techniques relying on the same type of polarization analysis could also be applied to the study of ultrafast dynamics in other research fields, such as femtochemistry [9]. 


\section{ACKNOWLEDGMENTS}

The H2020 funded Nanoscience Foundries and Fine Analysis (NNFA) project is acknowledged for the realization of an energy-dispersive intensity monitor, which will be described elsewhere. This work was partially supported by the European Research Council for the Advanced Grant X-Five (339128, PI:
V.M.). We warmly thank ANR for significant funding through the UMAMI project (ANR-15-CE24-0009-03). This work has also benefited from the help of Investissements d'Avenir of LabEx PALM (ANR-10-LABX-0039-PALM) through the XUV10 project. The authors finally acknowledge the financial support of Conseil Régional d'Ile-de-France, Contract No. 14014520.
[1] F. A. Weihe and P. H. Bucksbaum, J. Opt. Soc. Am. B 13, 157 (1996).

[2] P. Antoine, B. Carré, A. L'Huillier, and M. Lewenstein, Phys. Rev. A 55, 1314 (1997).

[3] X. Zhou, R. Lock, N. Wagner, W. Li, H. C. Kapteyn, and M. M. Murnane, Phys. Rev. Lett. 102, 073902 (2009).

[4] Y. Mairesse, J. Higuet, N. Dudovich, D. Shafir, B. Fabre, E. Mével, E. Constant, S. Patchkovskii, Z. Walters, M. Y. Ivanov, and O. Smirnova, Phys. Rev. Lett. 104, 213601 (2010).

[5] B. Vodungbo, A. Barszczak Sardinha, J. Gautier, G. Lambert, C. Valentin, M. Lozano, G. Iaquaniello, F. Delmotte, S. Sebban, J. Lüning, and P. Zeitoun, Opt. Express 19, 4346 (2011).

[6] A. Fleischer, O. Kfir, T. Diskin, P. Sidorenko, and O. Cohen, Nat. Photonics 8, 543 (2014).

[7] O. Kfir, P. Grychtol, E. Turgut, R. Knut, D. Zusin, D. Popmintchev, T. Popmintchev, H. Nembach, J. M. Shaw, A. Fleischer, H. Kapteyn, M. Murnane, and O. Cohen, Nat. Photonics 9, 99 (2014).

[8] F. Willems, C. T. L. Smeenk, N. Zhavoronkov, O. Kornilov, I. Radu, M. Schmidbauer, M. Hanke, C. von Korff Schmising, M. J. J. Vrakking, and S. Eisebitt, Phys. Rev. B 92, 220405(R) (2015).

[9] A. Ferré, C. Handschin, M. Dumergue, F. Burgy, A. Comby, D. Descamps, B. Fabre, G. A. Garcia, R. Géneaux, L. Merceron, E. Mével, L. Nahon, S. Petit, B. Pons, D. Staedter, S. Weber, T. Ruchon, V. Blanchet, and Y. Mairesse, Nat. Photonics 9, 93 (2014)

[10] G. Lambert, B. Vodungbo, J. Gautier, B. Mahieu, V. Malka, S. Sebban, P. Zeitoun, J. Luning, J. Perron, A. Andreev, S. Stremoukhov, F. Ardana-Lamas, A. Dax, C. P. Hauri, A. Sardinha, and M. Fajardo, Nat. Commun. 6, 6167 (2015).

[11] S. Stremoukhov, A. Andreev, B. Vodungbo, P. Salières, B. Mahieu, and G. Lambert, Phys. Rev. A 94, 013855 (2016).

[12] B. Mahieu, S. Stremoukhov, D. Gauthier, C. Spezzani, C. Alves, B. Vodungbo, P. Zeitoun, V. Malka, G. De Ninno, and G. Lambert, Phys. Rev. A 97, 043857 (2018).

[13] A. Kirilyuk, A. V. Kimel, and T. Rasing, Rev. Mod. Phys. 82, 2731 (2010).

[14] H.-C. Mertins, S. Valencia, D. Abramsohn, A. Gaupp, W. Gudat, and P. M. Oppeneer, Phys. Rev. B 69, 064407 (2004).

[15] S. Yamamoto, M. Taguchi, T. Someya, Y. Kubota, S. Ito, H. Wadati, M. Fujisawa, F. Capotondi, E. Pedersoli, M. Manfredda, L. Raimondi, M. Kiskinova, J. Fujii, P. Moras, T. Tsuyama, T. Nakamura, T. Kato, T. Higashide, S. Iwata, S. Yamamoto, S. Shin, and I. Matsuda, Rev. Sci. Instrum. 86, 083901 (2015).
[16] B. Vodungbo, A. Barszczak Sardinha, J. Gautier, G. Lambert, M. Lozano, S. Sebban, E. Meltchakov, F. Delmotte, V. LopezFlores, J. Arabski, C. Boeglin, E. Beaurepaire, R. Delaunay, J. Lüning, and P. Zeitoun, Europhys. Lett. 94, 54003 (2011).

[17] M. Ducousso, X. Ge, W. Boutu, D. Gauthier, B. Barbrel, F. Wang, A. Borta, A. Gonzalez, M. Billon, B. Vodungbo et al., Laser Phys. 24, 025301 (2014).

[18] C. La-O-Vorakiat, M. Siemens, M. M. Murnane, H. C. Kapteyn, S. Mathias, M. Aeschlimann, P. Grychtol, R. Adam, C. M. Schneider, J. M. Shaw, H. Nembach, and T. J. Silva, Phys. Rev. Lett. 103, 257402 (2009).

[19] B. Vodungbo, J. Gautier, G. Lambert, A. B. Sardinha, M. Lozano, S. Sebban, M. Ducousso, W. Boutu, K. Li, B. Tudu, M. Tortarolo, R. Hawaldar, R. Delaunay, V. López-Flores, J. Arabski, C. Boeglin, H. Merdji, P. Zeitoun, and J. Lüning, Nat. Commun. 3, 999 (2012).

[20] D. Rudolf, C. La-O-Vorakiat, M. Battiato, R. Adam, J. M. Shaw, E. Turgut, P. Maldonado, S. Mathias, P. Grychtol, H. T. Nembach, T. J. Silva, M. Aeschlimann, H. C. Kapteyn, M. M. Murnane, C. M. Schneider, and P. M. Oppeneer, Nat. Commun. 3, 1037 (2012).

[21] C. La-O-Vorakiat, E. Turgut, C. A. Teale, H. C. Kapteyn, M. M. Murnane, S. Mathias, M. Aeschlimann, C. M. Schneider, J. M. Shaw, H. T. Nembach, and T. J. Silva, Phys. Rev. X 2, 011005 (2012).

[22] S. Mathias, C. La-O-Vorakiat, P. Grychtol, P. Granitzka, E. Turgut, J. M. Shaw, R. Adam, H. T. Nembach, M. E. Siemens, S. Eich, C. M. Schneider, T. J. Silva, M. Aeschlimann, M. M. Murnane, and H. C. Kapteyn, Proc. Natl. Acad. Sci. U. S. A. 109, 4792 (2012).

[23] M. Hofherr, S. Moretti, J. Shim, S. Häuser, N. Y. Safonova, M. Stiehl, A. Ali, S. Sakshath, J. W. Kim, D. H. Kim, H. J. Kim, J. I. Hong, H. C. Kapteyn, M. M. Murnane, M. Cinchetti, D. Steil, S. Mathias, B. Stadtmüller, M. Albrecht, D. E. Kim, U. Nowak, M. Aeschlimann, Phys. Rev. B 98, 174419 (2018).

[24] B. Vodungbo, J. Gautier, G. Lambert, P. Zeitoun, and J. Lüning, Phys. Rev. X 3, 038001 (2013).

[25] B. Pfau, S. Schaffert, L. Müller, C. Gutt, A. Al-Shemmary, F. Büttner, R. Delaunay, S. Düsterer, S. Flewett, R. Frömter, J. Geilhufe, E. Guehrs, C. M. Günther, R. Hawaldar, M. Hille, N. Jaouen, A. Kobs, K. Li, J. Mohanty, H. Redlin, W. F. Schlotter, D. Stickler, R. Treusch, B. Vodungbo, M. Kläui, H. P. Oepen, J. Lüning, G. Grübel, and S. Eisebitt, Nat. Commun. 3, 1100 (2012).

[26] C. Boeglin, E. Beaurepaire, V. Halte, V. Lopez-Flores, C. Stamm, N. Pontius, H. A. Durr, and J.-Y. Bigot, Nature (London) 465, 458 (2010). 
[27] E. Jal, M. Makita, B. Rösner, C. David, F. Nolting, J. Raabe, T. Savchenko, A. el dine Kleibert, F. Capotondi, E. Pedersoli, L. Raimondi, M. Manfredda, I. Nikolov, X. Liu, A. el dine Merhe, N. Jaouen, J. Gorchon, G. Malinowski, M. Hehn, B. Vodungbo, and J. Lüning, Phys. Rev. B 99, 144305 (2019).

[28] J. Kuneš, P. M. Oppeneer, H.-C. Mertins, F. Schäfers, A. Gaupp, W. Gudat, and P. Novák, Phys. Rev. B 64, 174417 (2001).

[29] S. Valencia, A. Gaupp, W. Gudat, H. Mertins et al., New J. Phys. 8, 254 (2006).

[30] M. Elzo, E. Jal, O. Bunau, S. Grenier, Y. Joly, A. Ramos, H. Tolentino, J. Tonnerre, and N. Jaouen, J. Magn. Magn. Mater. 324, 105 (2012).

[31] F. Willems, S. Sharma, C. v. Korff Schmising, J. K. Dewhurst, L. Salemi, D. Schick, P. Hessing, C. Strüber, W. D. Engel, and S. Eisebitt, Phys. Rev. Lett. 122, 217202 (2019).

[32] B. Vodungbo, B. Tudu, J. Perron, R. Delaunay, L. Müller, M. H. Berntsen, G. Grübel, G. Malinowski, C. Weier, J. Gautier et al., Sci. Rep. 6, 18970 (2016).
[33] T. Ferté, N. Bergeard, L. Le Guyader, M. Hehn, G. Malinowski, E. Terrier, E. Otero, K. Holldack, N. Pontius, and C. Boeglin, Phys. Rev. B 96, 134303 (2017).

[34] L. Müller, C. Gutt, B. Pfau, S. Schaffert, J. Geilhufe, F. Büttner, J. Mohanty, S. Flewett, R. Treusch, S. Düsterer et al., Phys. Rev. Lett. 110, 234801 (2013).

[35] R. Gort, K. Bühlmann, S. Däster, G. Salvatella, N. Hartmann, Y. Zemp, S. Holenstein, C. Stieger, A. Fognini, T. U. Michlmayr, T. Bähler, A. Vaterlaus, and Y. Acremann, Phys. Rev. Lett. 121, 087206 (2018).

[36] K. C. Kuiper, T. Roth, A. J. Schellekens, O. Schmitt, B. Koopmans, M. Cinchetti, and M. Aeschlimann, Appl. Phys. Lett. 105, 202402 (2014).

[37] I. Radu, C. Stamm, A. Eschenlohr, F. Radu, R. Abrudan, K. Vahaplar, T. Kachel, N. Pontius, R. Mitzner, K. Holldack, A. Föhlisch, T. A. Ostler, J. H. Mentink, R. F. L. Evans, R. W. Chantrell, A. Tsukamoto, A. Itoh, A. Kirilyuk, A. V. Kimel, and T. Rasing, SPIN 5, 1550004 (2015).

[38] M. Buzzi, M. Makita, L. Howald, A. Kleibert, B. Vodungbo, P. Maldonado, J. Raabe, N. Jaouen, H. Redlin, K. Tiedtke et al., Sci. Rep. 7, 7253 (2017). 